\title{
A cytokinesis furrow is positioned by two consecutive signals
}

\author{
Henrik Bringmann ${ }^{1} \&$ Anthony A Hyman ${ }^{1}$
}

The position of the cytokinesis furrow in a cell determines the relative sizes of its two daughter cells as well as the distribution of their contents. In animal cells, the position of the cytokinesis furrow is specified by the position of the mitotic spindle ${ }^{1}$. The cytokinesis furrow bisects the spindle midway between the microtubule asters, at the site of the microtubule-based midzone, producing two daughter cells. Experiments in some cell types have suggested that the midzone positions the furrow ${ }^{2,3}$, but experiments in other cells have suggested that the asters position the furrow ${ }^{4,5}$. One possibility is that different organisms and cell types use different mechanisms to position the cytokinesis furrow. An alternative possibility is that both asters and the midzone contribute to furrow positioning ${ }^{6,7}$. Recent work in C. elegans has suggested that centrosome separation and the midzone are implicated in cytokinesis ${ }^{8}$. Here we examine the relative contributions of different parts of the mitotic spindle to positioning of the cytokinesis furrow in the C. elegans zygote. By spatially separating the spindle midzone from one of the asters using an ultraviolet laser, we show that the cytokinesis furrow is first positioned by a signal determined by microtubule asters, and then by a second signal that is derived from the spindle midzone. Thus, the position of the cytokinesis furrow is specified by two consecutive furrowing activities.

A mitotic spindle contains two structures implicated in cytokinesis furrow positioning: asters, which are radial arrays of centrosomenucleated microtubules, and the midzone, which forms between the separating chromatin at anaphase and consists of non-kinetochore spindle microtubules. A mitotic spindle is an inherently symmetric structure. A furrow-positioning cue from the asters would position the furrow midway between them. A midzone cue would position a furrow at the same place. In order to separate the contributions of the midzone and the asters to cytokinesis, the two structures must be spatially separated. In one-cell C. elegans embryos, the spindle forms in the middle of the cell at metaphase. At anaphase, cortical pulling forces pull on the microtubule asters, separating the two spindle poles ${ }^{9}$.

We took advantage of these pulling forces to design an experiment in which the position midway between the two asters was different from the position of the spindle midzone. An embryo was observed until the onset of anaphase (the time at which the midzone forms ${ }^{10}$ ). One aster was then separated from its associated chromatin using an ultraviolet laser, creating a cell with one isolated aster and the other aster still attached to the midzone. Cortical pulling forces moved the asters to opposite poles of the cell, positioning the spindle 'midzone' roughly one-third of the way along the aster-to-aster-distance (Fig. 1a-d and Supplementary Videos 1,2). Thus, the position of the midzone was different from the position midway between the two asters. We call this procedure asymmetric spindle severing (ASS). Following ASS, cytokinesis furrow ingression started midway between the asters. However, the furrow did not complete midway between the asters: a second furrow formed at the cell cortex closest to the midzone (Fig. 1e-g and Supplementary Videos 3-5). The two distinct furrows then met and cytokinesis completed. Thus, two furrows are observed after ASS, one between the asters and one directed towards the midzone. Both furrows contribute to the final position of the cleavage plane.

Using cylindrical cells, it has been shown that the mitotic apparatus can induce multiple furrows if it is successively displaced along the long axis of the cell ${ }^{11}$. A furrow can even be produced after removing the mitotic apparatus by aspiration before the onset of furrowing ${ }^{12}$. It could be that the spindle midzone specifies both furrows, one before and one after its displacement. Alternatively, the asters might specify the first furrow. To resolve this problem, we compared the effects of separating either the anterior or the posterior aster. The isolated aster moves further towards the poles of the cell compared with the aster that is still attached to the midzone. Thus, the position midway between the asters is different after anterior and posterior ASS. This difference is reflected in the difference of the position of the first furrow (see Fig. 2, the position of the first furrow is $53.7 \pm 0.9 \%$ along the embryo length after anterior ASS and $56.5 \pm 0.6 \%$ along the embryo length after posterior ASS; mean \pm s.e.m.; $P=0.041$ ). After ASS, the position of the first furrow is thus dependent on the position of the asters.

We also shifted the first furrow using monopolar spindles, as it has been shown that either an isolated aster or a monopolar spindle is sufficient to specify a cleavage plane $e^{4,13}$. To generate monopolar spindles, we repeated ASS and subsequently disintegrated the separated aster using the ultraviolet laser (Fig. 2 and Supplementary Videos 6, 7). Under these conditions, the first furrow was established further away from the remaining aster (ASS with intact aster, $12.8 \pm 0.2 \mu \mathrm{m}$; ASS followed by aster disintegration, $18.0 \pm 0.7 \mu \mathrm{m})$, at a position that was not related to the midzone position before ASS. The cleavage plane then corrected towards the midzone. The spatial shift of the first furrow away from the intact aster demonstrates that the furrow position is not defined before spindle severing, and is thus determined by microtubule asters. Taken together, these experiments show that the cytokinesis cleavage plane is specified by two consecutive signals derived from distinct components of the mitotic spindle: the microtubule asters and the spindle midzone.

Cleavage furrow formation is driven by actomyosin contraction, but little is known about the molecular mechanisms specifying where and when contraction will occur. To learn about the molecular control of redundant cleavage plane positioning, we assayed genes with known and potential roles in cytokinesis for their effects on the two furrows. Genes leading to defects in cytokinesis were chosen from the literature and genome-wide screens (see Supplementary Table 1). The gene products were depleted either by RNA interference (RNAi) or using established mutants, subjected to the ASS procedure, and analysed for the specification of the aster-positioned 
furrow (the first furrow), the specification of the midzone-positioned furrow (the second furrow), the ability to correct the aster-positioned furrow, and the extent of ingression of both furrows.

Here we describe four categories of cleavage furrow defects (Fig. 3 and Supplementary Videos 8-13). Supplementary Table 1 contains details of the genes tested. The first class consisted of rho-1, nmy-2, cyk-1, mlc-4, let-21, pfn-1 and act-4. In this class, neither furrow formed. Genes in this class were required for general aspects of actomyosin activity, and have previously been shown to prevent

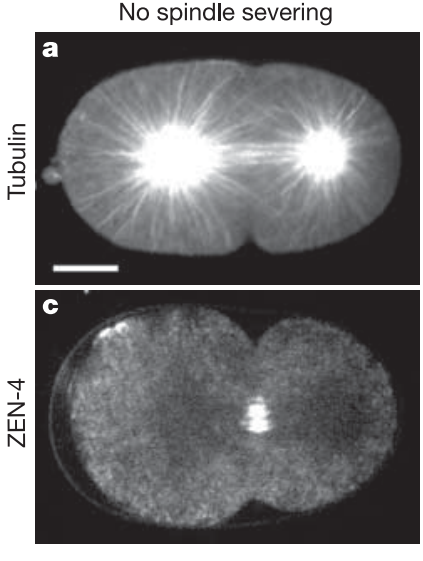

e

f
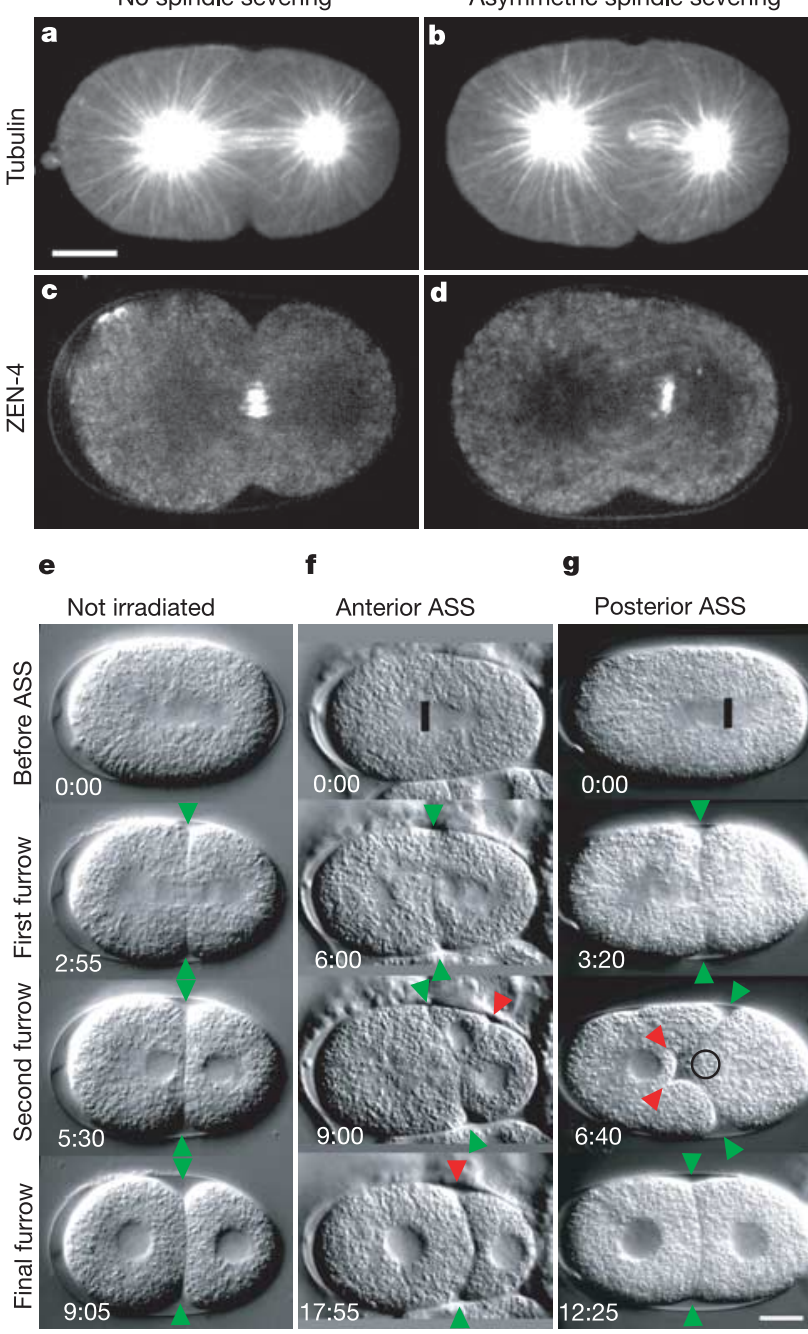

$\mathbf{g}$

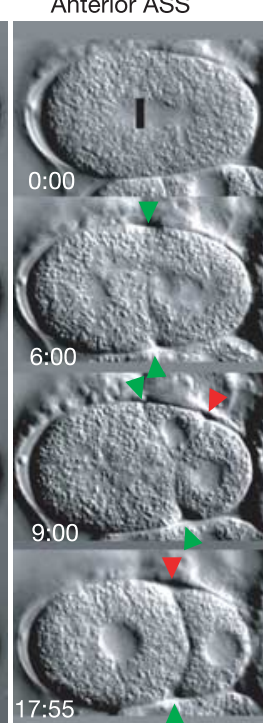

Posterior ASS

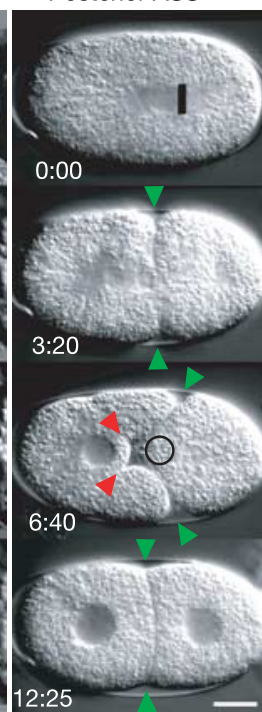

Figure 1 | Asymmetric spindle severing spatially separates the spindle midzone and the region midway between the asters, leading to the generation of two furrows. a-d, Alteration of spindle geometry. Spinningdisk microscopy images after ASS performed in a yellow fluorescent protein (YFP) $-\alpha$-tubulin background $(\mathbf{a}, \mathbf{b})$ or a zen-4-GFP background (c, d) to reveal the microtubule cytoskeleton and the spindle midzone, respectively. Unsevered control cells are shown in $\mathbf{a}$ and $\mathbf{c}$, severed spindles in $\mathbf{b}$ and $\mathbf{d}$. e-g, Generation of two furrows after ASS shown in DIC microscopy image series (compare Supplementary Videos 1-5). Time shown in minutes following ASS procedure. e, Wild-type embryo. f, Separation of the anterior aster. $\mathbf{g}$, Separation of the posterior aster. The irradiated region is indicated by a black bar. The posterior nucleus lies outside the focal plane in one image (position indicated by a black circle). Furrows often were unilateral after ASS. The asters seem to be attached to the ingressing furrow, which may cause the midzone to move off axis (see $\mathbf{f}$ ). First furrows are indicated in green, second furrows in red. The difference in furrowing after posterior and anterior ASS is probably due to the geometry of the cell. After anterior ASS, the midzone moves closer to the cortex compared with posterior ASS. In general, cytokinesis takes longer after ASS (roughly double the time compared to control). Posterior is to the right in a-g. Scale bars, $10 \mu \mathrm{m}$.

furrow formation ${ }^{14-16}$. We did not further analyse these genes. The second class consisted of air-2, zen-4 and cyk-4 (Fig. 3b). These genes have known roles in spindle midzone formation and completion of cytokinesis ${ }^{17-19}$. After ASS the aster-positioned furrow ingressed weakly compared with wild-type embryos. A midzone-positioned furrow did not form. The aster-positioned furrow regressed and cytokinesis failed. The weak ingression of the aster-positioned furrow indicates that these genes have general roles in furrow ingression.

The third class of genes leading to cleavage furrow defects consisted of $s p d-1$ and $k l p-7$ (Fig. 3c). Both of these genes are known to be required for midzone formation, but cytokinesis completes in one-cell embryos ${ }^{9,20}$. After ASS, embryos in this class
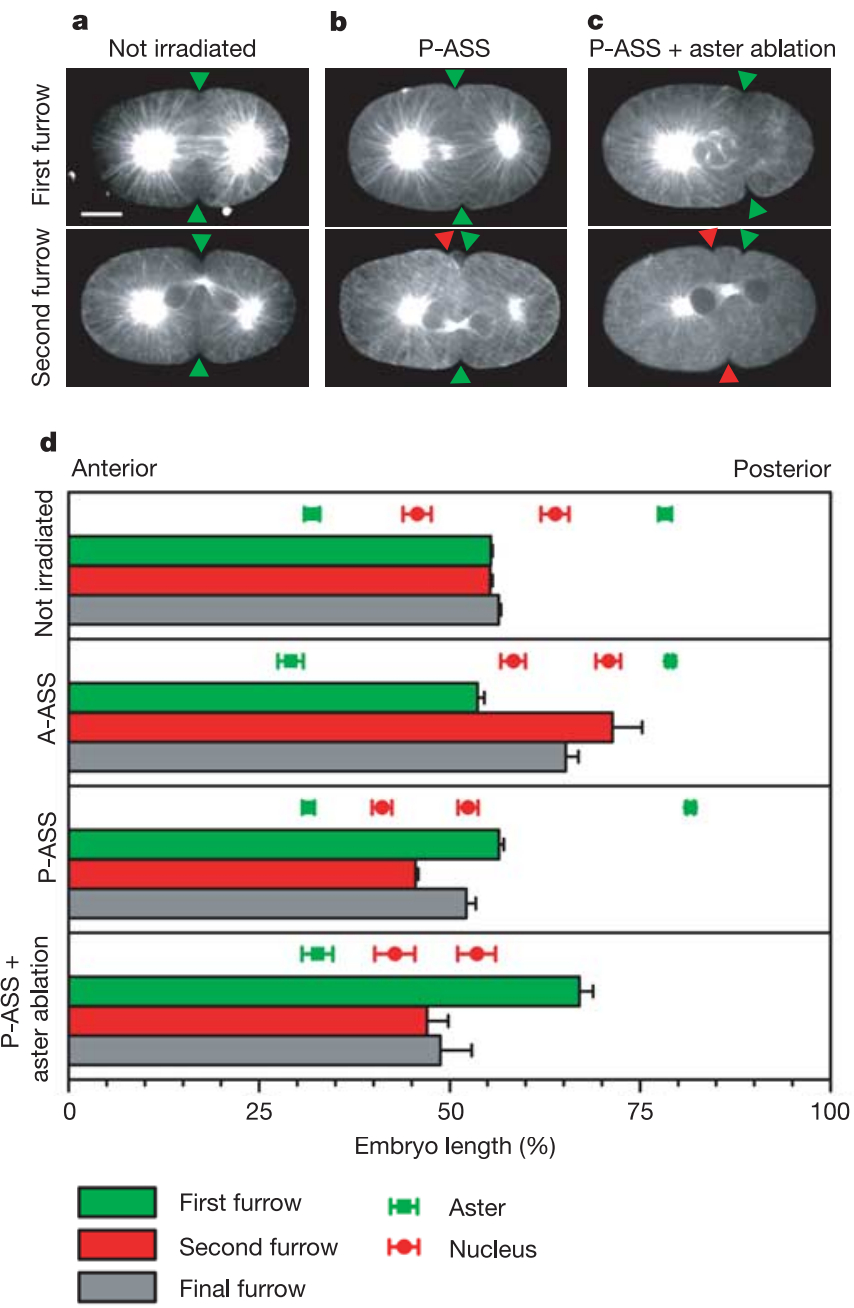

Figure 2 | Microtubule asters position the first cytokinesis furrow. The spindle midzone positions the second cytokinesis furrow. a-c, Spinning-disk microscopy images after posterior ASS (P-ASS) with (c) and without (b) subsequent aster ablation, observed by $\mathrm{YFP}-\alpha$-tubulin fluorescence. a, Unsevered control. Embryos in the top panels are shown at the point at which the first furrow (green arrowheads) ingresses. Compared with $\mathbf{a}$ and $\mathbf{b}$, the furrow in $\mathbf{c}$ is set up further away from the remaining aster. Embryos in the bottom panels are shown at the point at which the second furrow ingresses. The second furrow (red arrowheads in $\mathbf{b}$ and $\mathbf{c}$ ) always aims at the spindle midzone. Posterior is on the right. Scale bar, $10 \mu \mathrm{m}$. Compare Supplementary Videos 6 and 7 showing aster ablation in DIC microscopy. d, Quantification of spindle dimensions and furrow positions in unsevered embryos and embyros after anterior (A) and posterior (P) ASS, and P-ASS plus aster ablation. The position of the aster centres (green squares) and the nuclei (red dots) at the time the first furrow ingresses are indicated. The positions of the first (green), second (red) and final (grey) furrow are shown as histograms. Error bars are s.e.m. 
were capable of division but failed to segregate the DNA to the two daughter cells, yielding one cell with an aster and one cell with an aster and two nuclei. Progression of aster-positioned cleavage was fast compared with wild type $\left(6 \pm 1 \mu \mathrm{ms}^{-1}\right.$ in wild-type embryos and $11 \pm 1 \mu \mathrm{ms}^{-1}$ in spd-1(oj5) embryos), and the furrow did not
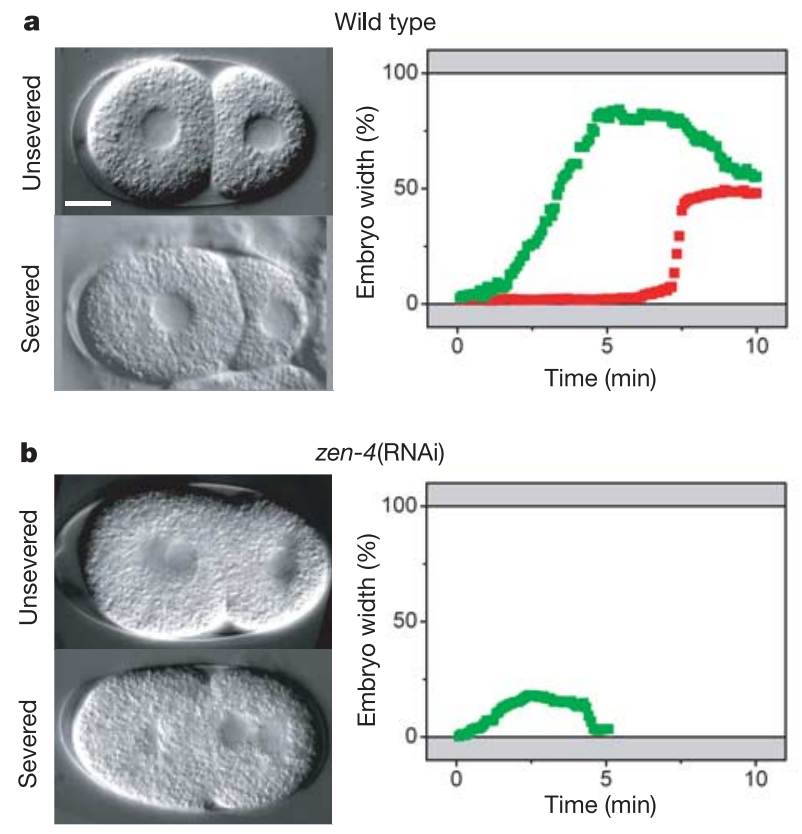

zen-4(RNAi)
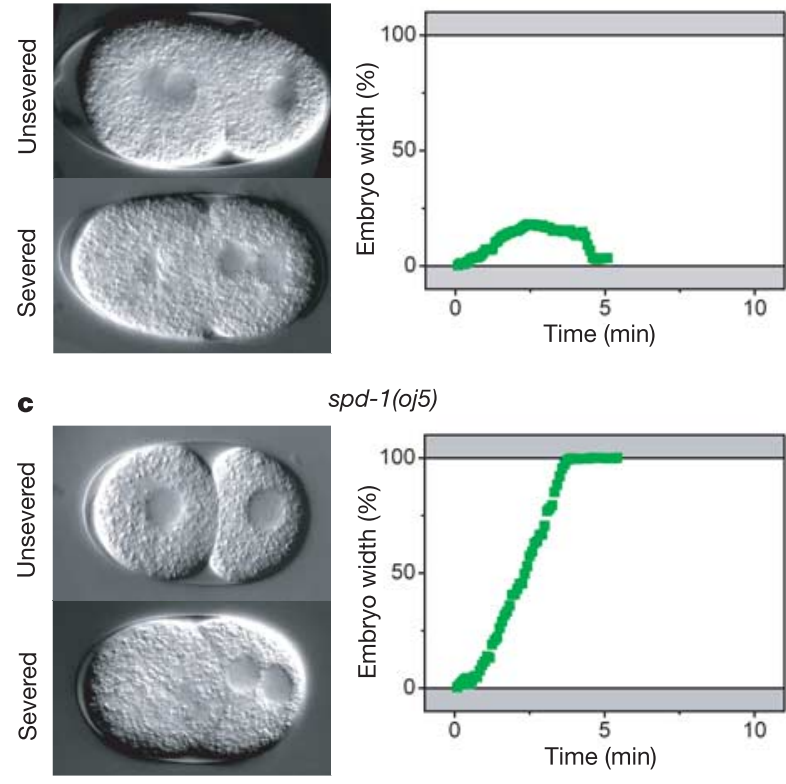

spd-1(oj5)
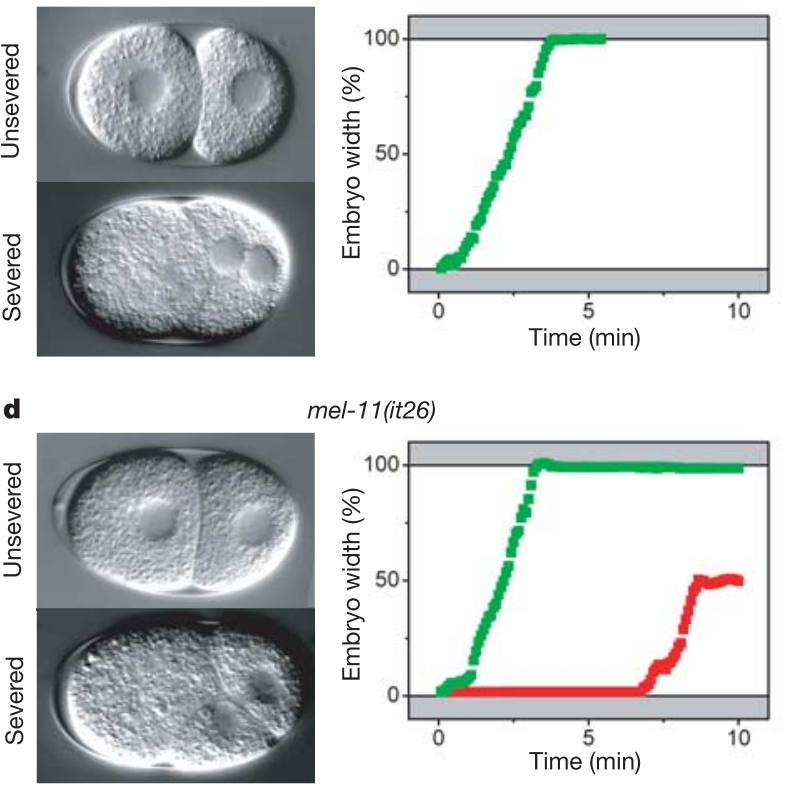

mel-11(it26)

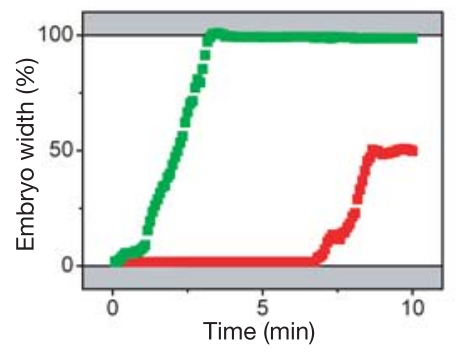

Figure 3 | Genetic control of redundant cleavage plane positioning. a-d, Phenotypic defects after ASS. Unsevered control and severed embryos are shown at the point at which the cytokinesis furrows are maximally ingressed. Right panels show cleavage progression over time, with the aster-positioned furrow in green and the midzone-positioned furrow in red. a, In wild-type embryos, the aster-positioned furrow does not complete, but instead pauses. The cytokinesis furrow position is then corrected by the midzone, and part of the aster-positioned furrow regresses. $\mathbf{b}$, Both furrowing activities are affected, leading to a failure in cytokinesis. c, No correction mechanism is present; the embryo divides solely using its aster-based mechanism, resulting in one cell that contains no nucleus, and one cell that contains two nuclei. d, Failure to correct the aster-positioned furrow means that the aster-positioned furrow completes before the midzone-positioned furrow starts, leading to the generation of three cells. pause, suggesting that the spindle midzone inhibits the asterpositioned furrow. The absence of a midzone-positioned furrow indicates that the defective spindle midzone in $s p d-1$ and $k l p-7$ mutants is not able to provide a positional cue. Additionally, the completion of cytokinesis in spd-1 and klp-7 mutants solely on the basis of an aster-positioned furrow demonstrates that cytokinesis can complete without a functional midzone, confirming previous studies $^{12,20}$. Cytokinesis in $s p d-1$ and $k l p-7$ mutants highlights the redundancy of the cleavage furrow specification mechanism. This experiment further shows that the two furrow-positioning mechanisms are genetically separable.

The fourth class consisted of one gene, mel-11, which is a myosin light chain phosphatase that inhibits non-muscle myosin (NMY-2) contractility (Fig. 3d). In mel-11 embryos, cytokinesis occurs twice as fast as in wild-type embryos ${ }^{21}$. In this class, the aster-positioned furrow ingressed and completed. However, a midzone-dependent furrow was also specified and completed, resulting three cells: one containing an aster, one containing a nucleus, and one containing an aster and a nucleus. We measured the progression of aster-dependent cleavage as $6 \pm 1 \mu \mathrm{m} \mathrm{s}^{-1}$ in wild-type embryos and $11 \pm 2 \mu \mathrm{m} \mathrm{s}^{-1}$ in mel-11(it26) embryos. Thus, reduced ingression of the asterpositioned furrow in the presence of a spindle midzone is probably regulated through myosin activity, and facilitates a positional correction mechanism by the spindle midzone.

By physically changing the geometry of the mitotic apparatus in a C. elegans embryo at the one-cell stage, we have shown that the position of the cytokinesis furrow is controlled by the consecutive and concerted action of two signals. The first positional signal is provided by microtubule asters. This is followed by a second signal specified by the spindle midzone. The first, aster-derived signal alone seems to be sufficient for cleavage. The second signal can probably also cleave the cell alone, as the midzone can position contractile material and induce a furrow after ASS. The second signal provides a robust correction mechanism to ensure that the genetic material is segregated equally to the two daughter cells. The spindle midzone is not required for completion and abscission in our experiments. However, the spindle midzone negatively influences the ingression of the aster-positioned furrow, probably by regulating myosin activity, perhaps through MEL-11. It facilitates the correction mechanism by the spindle midzone. The dominance of the midzone-positioned furrow is further highlighted by a persistent flow of green fluorescent protein (GFP)-tagged myosin (NMY-2-GFP) patches into the midzone-positioned furrow, while flow into the aster-positioned furrow ceases (Supplementary Videos 14 and 15). Redundant cytokinesis signals could be a universal mechanism to increase the precision with which the cleavage plane is positioned. Different cell types, however, might use the two mechanisms to different extents.

\section{METHODS}

Laser setup, microscopy and image analysis. The laser ablation experiments were performed using a highly focused ultraviolet laser beam and observed using differential interference contrast (DIC) and spinning-disk microscopy as described $^{22}$, except that a Melles Griot ArIon Laser $(488 \mathrm{~nm}, 100 \mathrm{~mW})$ laser was used for fluorescence imaging. All ablations were performed in DIC optics. Quantification of spindle dimensions and furrow positions were made using Scion Image. Cytokinesis furrows were tracked in DIC movies using Metamorph software. All errors are reported as s.e.m. Figs were prepared using Photoshop and Illustrator (Adobe).

Asymmetric spindle severing (ASS). Asymmetric spindle severing was performed just after anaphase onset (identified by the disappearance of the metaphase plate) and observed by DIC microscopy. At the time of the spindle severing, spindle midzone components like ZEN-4 are already localized to the spindle midzone (unpublished observations using ZEN-4-GFP). Five to ten shots were fired at the region midway between one aster centre and the separated chromatin (see Supplementary Videos 1 and 2). One shot consisted of 25 optical pulses at a repetition rate of $250 \mathrm{~Hz}$. If the same number of shots was fired in the region between spindle and cortex, no severing of the spindle is observed (data 
not shown). The position of the first furrow was measured at the point at which it had ingressed roughly two-thirds of the egg width. The position of the second furrow was measured at the first point at which the furrow was visible. If the furrow showed bent growth, we measured the final position to which the furrow was aiming. The position of the final furrow was measured $(n=4)$ at the point at which the two blastomeres rounded up and the furrow was perpendicular to the anterior-posterior axis of the embryo. For furrow tracking $(n=3)$, we focused such that the largest opening of the contractile ring was visible. We compared the tracking data for an NMY-2-GFP embryo when collected by DIC or fluorescence microscopy and obtained comparable results. For simplicity, DIC videos were used for all quantifications. After ASS, the aster-positioned furrow is normally unilateral in wild-type embryos but bilateral in MEL-11 and SPD-1 embryos, which accounts for the different cytokinesis progression speeds.

Aster ablation. In order to ablate the asters, 15-20 additional shots were delivered in the centre of the aster (the region devoid of yolk granules) after it had been isolated from the mitotic spindle. The microscope stage was rapidly moved in circles around the centre of the aster during shooting to prevent spreading of the aster fragments (Supplementary Video 6). If the shots were distributed in the astral region containing yolk granules, the aster remained intact and the first furrow was not displaced (data not shown). Optical sections were taken with a spinning-disk microscope. We scanned through the embryo to verify the absence of the aster. Furrow positions were quantified (as for ASS) for five embryos. Using DIC and spinning-disk microscopy, we observed the cell containing the irradiated aster for a time equivalent to several additional cell division cycles, and did not see any sign of an aster reforming.

Worm culture, strains, alleles and RNAi. C. elegans were cultured as described ${ }^{9}$. All RNAi-mediated depletions were performed in the RNAi hypersensitive strains NL2099 (ref. 23) or GR1373 (ref. 24). Both strains behaved like wildtype N2 worms after ASS. RNAi was performed either by injection of doublestranded RNA or by feeding, as described ${ }^{16,25}$. Worms were analysed 48 or $72 \mathrm{~h}$ after injection or feeding, respectively. For feeding experiments, worms were mated with males to maintain egg-laying. Mutants were shifted to a restrictive temperature $5-24 \mathrm{~h}$ before analysis. For a list of mutants and RNAi methods used, see Supplementary Table 1. Genes were classified as wild type if the embryos displayed the published phenotype and if, under those conditions, two furrows were observed. Typically, 5-10 experiments were performed for each gene. All DIC experiments were carried out at $23^{\circ} \mathrm{C}$, spinning-disk data were collected at $17^{\circ} \mathrm{C}$.

\section{Received 7 February; accepted 16 May 2005.}

1. Rappaport, R. Cytokinesis in Animal Cells (Cambridge Univ. Press, Cambridge, 1996).

2. Bonaccorsi, S., Giansanti, M. G. \& Gatti, M. Spindle self-organization and cytokinesis during male meiosis in asterless mutants of Drosophila melanogaster. J. Cell Biol. 142, 751-761 (1998)

3. Cao, L. G. \& Wang, Y. L. Signals from the spindle midzone are required for the stimulation of cytokinesis in cultured epithelial cells. Mol. Biol. Cell 7, 225-232 (1996).

4. Rappaport, R. \& Rappaport, B. N. Surface contractile activity associated with isolated asters in cylindrical sand dollar eggs. J. Exp. Zool. 235, 217-226 (1985).

5. Rappaport, R. Experiments concerning the cleavage stimulus in sand dollar eggs. J. Exp. Zool. 148, 81-89 (1961)

6. Wang, Y. L. The mechanism of cytokinesis: reconsideration and reconciliation. Cell Struct. Funct. 26, 633-638 (2001)

7. Canman, J. C. \& Wells, W. A. Rappaport furrows on our minds: the ASCB Cytokinesis Meeting Burlington, VT July 22-25, 2004. J Cell Biol 166, 943-948 (2004).

8. Dechant, R. \& Glotzer, M. Centrosome separation and central spindle assembly act in redundant pathways that regulate microtubule density and trigger cleavage furrow formation. Dev. Cell 4, 333-344 (2003).

9. Grill, S. W., Gonczy, P., Stelzer, E. H. \& Hyman, A. A. Polarity controls forces governing asymmetric spindle positioning in the Caenorhabditis elegans embryo. Nature 409, 630-633 (2001)

10. Mishima, M., Pavicic, V., Gruneberg, U., Nigg, E. A. \& Glotzer, M. Cell cycle regulation of central spindle assembly. Nature 430, 908-913 (2004).

11. Rappaport, R. Repeated furrow formation from a single mitotic apparatus in cylindrical sand dollar eggs. J. Exp. Zool. 234, 167-171 (1985).

12. Hiramoto, Y. Cell division without mitotic apparatus in sea urchin eggs. Exp. Cell Res. 11, 630-636 (1956)

13. Canman, J. C. et al. Determining the position of the cell division plane. Nature 424, 1074-1078 (2003).

14. Shelton, C. A., Carter, J. C., Ellis, G. C. \& Bowerman, B. The nonmuscle myosin regulatory light chain gene $\mathrm{mlc}-4$ is required for cytokinesis, anterior-posterior polarity, and body morphology during Caenorhabditis elegans embryogenesis. J. Cell Biol. 146, 439-451 (1999).

15. Severson, A. F., Baillie, D. L. \& Bowerman, B. A Formin Homology protein and a profilin are required for cytokinesis and Arp2/3-independent assembly of cortical microfilaments in C. elegans. Curr. Biol. 12, 2066-2075 (2002).

16. Sonnichsen, B. et al. Full-genome RNAi profiling of early embryogenesis in Caenorhabditis elegans. Nature 434, 462-469 (2005)

17. Schumacher, J. M., Golden, A. \& Donovan, P. J. AIR-2: An Aurora/Ipl1-related protein kinase associated with chromosomes and midbody microtubules is required for polar body extrusion and cytokinesis in Caenorhabditis elegans embryos. J. Cell Biol. 143, 1635-1646 (1998).

18. Raich, W. B., Moran, A. N., Rothman, J. H. \& Hardin, J. Cytokinesis and midzone microtubule organization in Caenorhabditis elegans require the kinesinlike protein ZEN-4. Mol. Biol. Cell 9, 2037-2049 (1998).

19. Jantsch-Plunger, V. et al. CYK-4: A Rho family GTPase activating protein (GAP) required for central spindle formation and cytokinesis. J. Cell Biol. 149, 1391-1404 (2000).

20. Verbrugghe, K. J. \& White, J. G. SPD-1 is required for the formation of the spindle midzone but is not essential for the completion of cytokinesis in C. elegans embryos. Curr. Biol. 14, 1755-1760 (2004)

21. Piekny, A. J. \& Mains, P. E. Rho-binding kinase (LET-502) and myosin phosphatase (MEL-11) regulate cytokinesis in the early Caenorhabditis elegans embryo. J. Cell Sci. 115, 2271-2282 (2002).

22. Grill, S. W., Howard, J., Schaffer, E., Stelzer, E. H. \& Hyman, A. A. The distribution of active force generators controls mitotic spindle position. Science 301, 518-521 (2003).

23. Simmer, F. et al. Loss of the putative RNA-directed RNA polymerase RRF-3 makes C. elegans hypersensitive to RNAi. Curr. Biol. 12, 1317-1319 (2002).

24. Kennedy, S., Wang, D. \& Ruvkun, G. A conserved siRNA-degrading RNase negatively regulates RNA interference in C. elegans. Nature 427, 645-649 (2004)

25. Kamath, R. S. et al. Systematic functional analysis of the Caenorhabditis elegans genome using RNAi. Nature 421, 231-237 (2003).

Supplementary Information is linked to the online version of the paper at www.nature.com/nature.

Acknowledgements We thank the Caenorhabditis Genetics Center, the UK HGMP Resource Centre, Cenix Bioscience, T. Stiernagle, J. Ahringer, M. Glotzer, M. Srayko, C. Cowan, L. Pelletier, P. E. Mains, A. Schwager and A. Pozniakovskiy for reagents; C. Cowan, S. Grill, S. Schoenegg, J. Stamford and M. Srayko for advice and help on the experimental setup; and C. Cowan, J. Howard and K. Verbrugghe for comments on the manuscript. H.B. was supported by a predoctoral fellowship from the Boehringer Ingelheim Foundation.

Author Information Reprints and permissions information is available at npg.nature.com/reprintsandpermissions. The authors declare no competing financial interests. Correspondence and requests for materials should be addressed to H.B. (bringman@mpi-cbg.de). 